\title{
RDL mutations predict multiple insecticide resistance in Anopheles sinensis in Guangxi, China
}

Chan Yang ${ }^{1,2}$, Zushi Huang $^{1}$, Mei Li ${ }^{1}$, Xiangyang Feng ${ }^{3}$ and Xinghui Qiu ${ }^{1 *} \mathbb{0}$

\begin{abstract}
Background: Anopheles sinensis is a major vector of malaria in China. The gamma-aminobutyric acid (GABA)-gated chloride channel, encoded by the RDL (Resistant to dieldrin) gene, is the important target for insecticides of widely varied structures. The use of various insecticides in agriculture and vector control has inevitably led to the development of insecticide resistance, which may reduce the control effectiveness. Therefore, it is important to investigate the presence and distribution frequency of the resistance related mutation(s) in An. sinensis RDL to predict resistance to both the withdrawn cyclodienes (e.g. dieldrin) and currently used insecticides, such as fipronil.

Methods: Two hundred and forty adults of An. sinensis collected from nine locations across Guangxi Zhuang Autonomous Region were used. Two fragments of An. sinensis RDL (AsRDL) gene, covering the putative insecticide resistance related sites, were sequenced respectively. The haplotypes of each individual were reconstructed by the PHASE2.1 software, and confirmed by clone sequencing. The phylogenetic tree was built using maximum-likelihood and Bayesian inference methods. Genealogical relations among different haplotypes were also analysed using Network 5.0.

Results: The coding region of AsRDL gene was 1674 bp long, encoding a protein of 557 amino acids. AsRDL had 98.0\% amino acid identity to that from Anopheles funestus, and shared common structural features of Cys-loop ligandgated ion channels. Three resistance-related amino acid substitutions (A296S, V327I and T345S) were detected in all the nine populations of An. sinensis in Guangxi, with the 2965 mutation being the most abundant (77-100\%), followed by $345 S$ (22-47\%) and 327I (8-60\%). 38 AsRDL haplotypes were identified from 240 individuals at frequencies ranging from 0.2 to $34.8 \%$. Genealogical analysis suggested multiple origins of the $345 \mathrm{~S}$ mutation in AsRDL.
\end{abstract}

Conclusions: The near fixation of the 2965 mutation and the occurrence of the 327 and 3455 mutations in addition to 296S, in all the nine tested An. sinensis populations in Guangxi, strongly indicate a risk of multiple insecticide resistance. The haplotype diversity plus genetic heterogeneities in the geographical distribution, and multiple origins of AsRDL alleles call for a location-customized strategy for monitoring and management of insecticide resistance.

Keywords: Anopheles sinensis, Haplotype, Genealogical analysis, Guangxi Zhuang Autonomous Region, Gammaaminobutyric acid gated chloride channel

\section{Background}

Guangxi Zhuang Autonomous Region was once a malaria-endemic region with more than 5 million

\footnotetext{
*Correspondence: qiuxh@ioz.ac.cn

1 State Key Laboratory of Integrated Management of Pest Insects and Rodents, Institute of Zoology, Chinese Academy of Sciences, Beijing 100101, China

Full list of author information is available at the end of the article
}

malaria patients per year being recorded before 1949 [1]. After continued efforts, particularly the "National Malaria Control Programme", the "Basically Eliminating Malaria" and the "Action Plan of Malaria Elimination (2010-2020)" [2, 3], the malaria burden has been substantially reduced in Guangxi [4]. However, the risk of malaria re-emergence remains, because many imported malaria cases have been reported due to ever increasing cross-border population migration, and the natural 
environment in Guangxi, consisting of rice fields, provides many mosquito breeding sites [3-6].

Chemical control of vectors has played an important role in malaria control and elimination [7]. In China, Anopheles sinensis is a major vector of malaria. The use of insecticides in the control of An. sinensis itself, and in agriculture, has inevitably led to increasing insecticide resistance in Chinese An. sinensis [8-10]. The development of insecticide resistance can reduce the effectiveness of vector control, therefore, understanding the levels of resistance and mechanisms responsible for this resistance is a core concept to effectively manage An. sinensis. However, the insecticide resistance issue in An. sinensis has so far received little attention. Although the distribution and frequency of insecticide resistance-conferring mutations in the acetylcholinesterase and voltage gated sodium channel were determined in An. sinensis collected extensively across Guangxi in previous studies $[11,12]$, other questions related to insecticide resistance remain to be answered.

The insect gamma-aminobutyric acid (GABA) receptor subunit encoded by the $R D L$ (Resistant to dieldrin) gene plays a central role in neuronal signaling and is involved in various processes [13]. RDL has been the target for insecticides of various chemical structures such as cyclodienes and fipronil [14]. RDL is also a potential secondary target for neonicotinoids and pyrethroids [13]. Previous studies have documented that mutations in RDL are associated with insecticide resistance in multiple insect species. For example, the replacement of a single alanine (Ala301 in Drosophila) at the start of transmembrane segment M2 with either serine or glycine makes the Drosophila strains resistant to dieldrin [15]. Similarly, the A296S substitution (analogous to position 301 in Drosophila melanogaster) was associated with dieldrin resistance in mosquitoes such as Anopheles arabiensis, Anopheles funestus, Anopheles stephensi, Aedes aegypti, Aedes albopictus and Culex pipiens quinquefasciatus [16-19]. Another replacement of the equivalent residue (A301N) was observed in the fipronil-resistant planthopper Laodelphax striatellus [20, 21]. Besides mutation at residue 296, a substitution (V327I) was reported on a background of $296 \mathrm{~S}$ in dieldrin-resistant An. funestus [18]. In addition, two different substitutions at residue 350 (T350M and T350S) were observed in Drosophila linked with 301G [22] and 301S [23], respectively. Recently, a second RDL mutation (R300Q) in combination with A302S was found to be associated with much higher levels of fipronil resistance (237-fold) in Nilaparvata lugens [24].

Until now, there is no published report about RDL in the malaria vector $A n$. sinensis. In this study, the $R D L$ gene in $A n$. sinensis (AsRDL) was identified, and the naturally occurring genetic mutations in $A s R D L$ and their geographical distribution in Guangxi were investigated, in the hope of predicting their both historical role in cyclodiene (e.g. dieldrin) resistance and potential influence in conferring resistance to currently used insecticides such as fipronil.

\section{Methods \\ Samples}

Anopheles sinensis adults used in the study were caught by light trap (wave length $365 \mathrm{~nm}$ ) from July to September in 2014, around farmers' houses at different geographical locations across Guangxi. The sampling sites were part of the malaria vector monitoring sites that were set up partly because there were imported malaria cases observed since 2010 in these regions. A brief description of the sampling sites was provided previously [12].

Anopheles sinensis individual mosquitoes were morphologically identified [25], and put into 100- $\mu$ l Eppendorf tubes containing $100 \%$ ethanol solution at $4{ }^{\circ} \mathrm{C}$. The accuracy of morphological classification was confirmed by molecular detection of ten randomly selected adults from each population using the rDNA-ITS2 method [26].

\section{Sequencing of the full length $R D L$ coding sequence from Anopheles sinensis}

Total RNA was extracted from ten adults of a laboratory strain of An. sinensis [27] by using TRIzol (Invitrogen, CA, USA) according to the manufacturer's protocol. First-strand cDNA was synthesized from total RNA (1 $\mu \mathrm{g})$ using PrimeScript First Strand Synthesis Kit according to the manufacturer's instructions (Takara, Dalian, China). The full length open reading frame sequence of the GABA-gated chloride channel gene was amplified by PCR using the primers (AsRDLfullcd-F:ATGTCGCTAACCATCGAAGTTCCGC; AsRDLfullcd-R: TTACTTATCCTCACCGAGCAGCA) commercially synthesized by Invitrogen (China). The PCR mixture $(50 \mu \mathrm{l})$ consisted of $2 \mu \mathrm{l}$ cDNA template, $1 \mu \mathrm{l}$ PrimeStar $^{\circledR}$ GXL (Takara), $10 \mu \mathrm{l} 5 \times$ Buffer, $4 \mu \mathrm{l}$ $2.5 \mathrm{mM}$ dNTPs, $1 \mu \mathrm{l} 10 \mathrm{mM}$ each primer, and $31 \mu \mathrm{l}$ $\mathrm{ddH}_{2} \mathrm{O}$. The thermal cycling profile consisted of an initial step of denaturation at $95{ }^{\circ} \mathrm{C}$ for $3 \mathrm{~min}$; followed by 36 cycles of $98{ }^{\circ} \mathrm{C}$ for $10 \mathrm{~s}, 55^{\circ} \mathrm{C}$ for $15 \mathrm{~s}, 68^{\circ} \mathrm{C}$ for $2 \mathrm{~min}$; and a final extension step at $68^{\circ} \mathrm{C}$ for $10 \mathrm{~min}$. The PCR products were gel purified (Takara, Dalian, China) and then subcloned into pEasy-T1 and transformed the Escherichia coli Trans $5 \alpha$ strain (Transgen, Beijing, China). The nucleotide sequences of $A s-R D L$ gene were identified by direct sequencing of PCR products and/or clone sequencing from two directions. 


\section{PCR amplification of AsRDL fragments}

The genomic DNA of individual adult mosquitoes was prepared as described elsewhere [11, 28]. A fragment of exon 7 encoding the M2 transmembrane domain region was amplified using primers ASRdl-7F (AGTTTGTACGTTCGATGGGTTA) and ASRdl-7R (CCAGCAGACTGGCAAATACC). Another fragment containing partial intron 7 and exon 8 was obtained using primers ASRdl-8F (GCGTGGCACTGATATCGTTT) and ASRdl8R (CCTTGCCGGGATGTATCAGA). The PCR mixture $(20 \mu \mathrm{l})$ consisted of $5 \mu \mathrm{l}$ DNA template, $0.5 \mu \mathrm{l}$ Takara Taq Polymerase, $2 \mu \mathrm{l} 10 \times$ Taq Buffer, $2 \mu \mathrm{l} 2.5 \mathrm{mM}$ dNTPs,

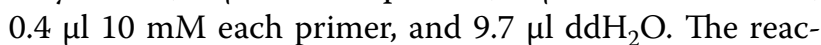
tion program was $95{ }^{\circ} \mathrm{C}$ for $5 \mathrm{~min}$, followed by 38 cycles each with $95{ }^{\circ} \mathrm{C}$ for $30 \mathrm{~s}, 55{ }^{\circ} \mathrm{C}$ for $30 \mathrm{~s}, 72{ }^{\circ} \mathrm{C}$ for $30 \mathrm{~s}$, and by a final extension of $10 \mathrm{~min}$ at $72{ }^{\circ} \mathrm{C}$. PCR products were gel-purified and sequenced using ASRdl-7F and ASRdl-8R, respectively (BGI, China).

\section{Haplotype reconstruction and confirmation}

Genotype data were carefully inspected by using Chromas 2.01 (Technelysium Pty Ltd, Australia). AsRDL haplotypes were reconstructed by software PHASE2.1 [29]. The presence (and accuracy) of the haplotypes that were reconstructed by PHASE in heterozygotes was confirmed by sequencing of three to five clones.

\section{Genealogical analysis}

The AsRDL haplotypes were used to build the phylogenetic topologies by maximum-likelihood (ML) and Bayesian inference (BI) methods. The best-fitting substitution model was determined under the Bayesian information criteria (BIC) using jModelTest 2.1.1 [30]. The best-fitting model of nucleotide substitution for $A s R D L$ fragment was TVM+I. PhyML 3.0 was used to perform ML analyses [31]. The non-parametric bootstrap with 1000 replicates was used to estimate the support for each internal branch of the ML phylogeny. The program MrBayes 3.2.1 was used for Bayesian phylogenetic inference [32]. Markov chains were run for $10^{7}$ generations with two independent runs, and trees were sampled every 1000 generations. The initial $25 \%$ of trees were discarded as burn-in, and the remaining trees $(15,002)$ were used to estimate the majority-rule consensus tree and the Bayesian posterior probabilities (BPP). Genealogical relations among different haplotypes were also analysed using Network 5.0 [33].

\section{Results}

\section{Identification of $A s R D L$ gene}

The coding region of $A s R D L$ gene was 1674 bp long, encoding a protein of 557 amino acids. Four non-synonymous variations that result in amino acid substitutions
(R119G, I162V, I176V, I278V) were identified in cDNA sequences (Fig. 1). Mapping the coding region to the genomic sequence (KE525297.1) showed that the AsRDL gene consists of nine exons and eight introns (Fig. 2).

Alignment analysis (Fig. 3) showed that AsRDL was highly similar to that from Anopheles funestus (98.0\% amino acid identity), followed by Anopheles gambiae (97.9\%). A lower identity (77.3\%) was determined when compared with Drosophila melanogaster RDL. AsRDL had the common structural features shared by known Cys-loop ligand-gated ion channels (LGICs), including the well-conserved dicysteine-loop structure, a large extracellular domain and four hydrophobic transmembrane regions (TM 1-4) (Fig. 3).

\section{Identification of naturally occurring genetic mutations in $A s R D L$}

A $184 \mathrm{bp}$-in-length sequence of partial exon 7 (namely $7 \mathrm{~F}$ ), and a $494 \mathrm{bp}$ region containing $81 \mathrm{bp}$ in intron 7 and $413 \mathrm{bp}$ in exon 8 (namely $8 \mathrm{R}$ ), were obtained from 240 individuals, respectively. Three polymorphic sites were observed in 7F (Fig. 4). From the 8R sequences, ten and eighteen polymorphic sites were identified in intron 7 and exon 8, respectively (Fig. 4).

From these sequencing data, 12 non-synonymous polymorphic sites were recorded. Two non-synonymous mutations within exon 7 were identified in the first nucleotide (T/G) of codon 296 and in the first nucleotide (A/G) of codon 327, which lead to A296S and V327I substitutions, respectively (Fig. 5). Ten non-synonymous mutations were observed within exon 8 , which result in 345T/S, 349L/M, 356R/G, 357K/L/M/N, 360F/L, 408G/H and 473T/A substitutions, respectively (Fig. 1).

The distribution frequencies of these substitutions were presented in Table 1. Among these substitutions, $356 \mathrm{G}, 357 \mathrm{~L}, 357 \mathrm{~N}$ and $408 \mathrm{H}$ were detected in only one population at a very low frequency. In contrast, three putative resistance-related substitutions (A296S, V327I and T345S) were widely distributed with relatively higher frequencies (Table 1 and Fig. 6).

\section{Distribution and frequency of resistance-related mutations} The $296 \mathrm{~S}$ mutation was the most abundant (77-100\%), followed by the 345S (22-47\%) and 327I (8-60\%) mutations. Notably, the resistant allele (296S) had $100 \%$ frequency in six of the nine populations (Table 1).

Based on the genotypes of the three resistance-related sites, eleven combinations were observed (Table 2). Most individuals carried at least one resistant mutation (combination 1-10). A few individuals from Baise (6.8\%) and Liuzhou (12.5\%) were susceptible homozygotes (combination 11). Two combinations (2 and 3 ) contained mutations in all the three positions. Notably, either 327I or 


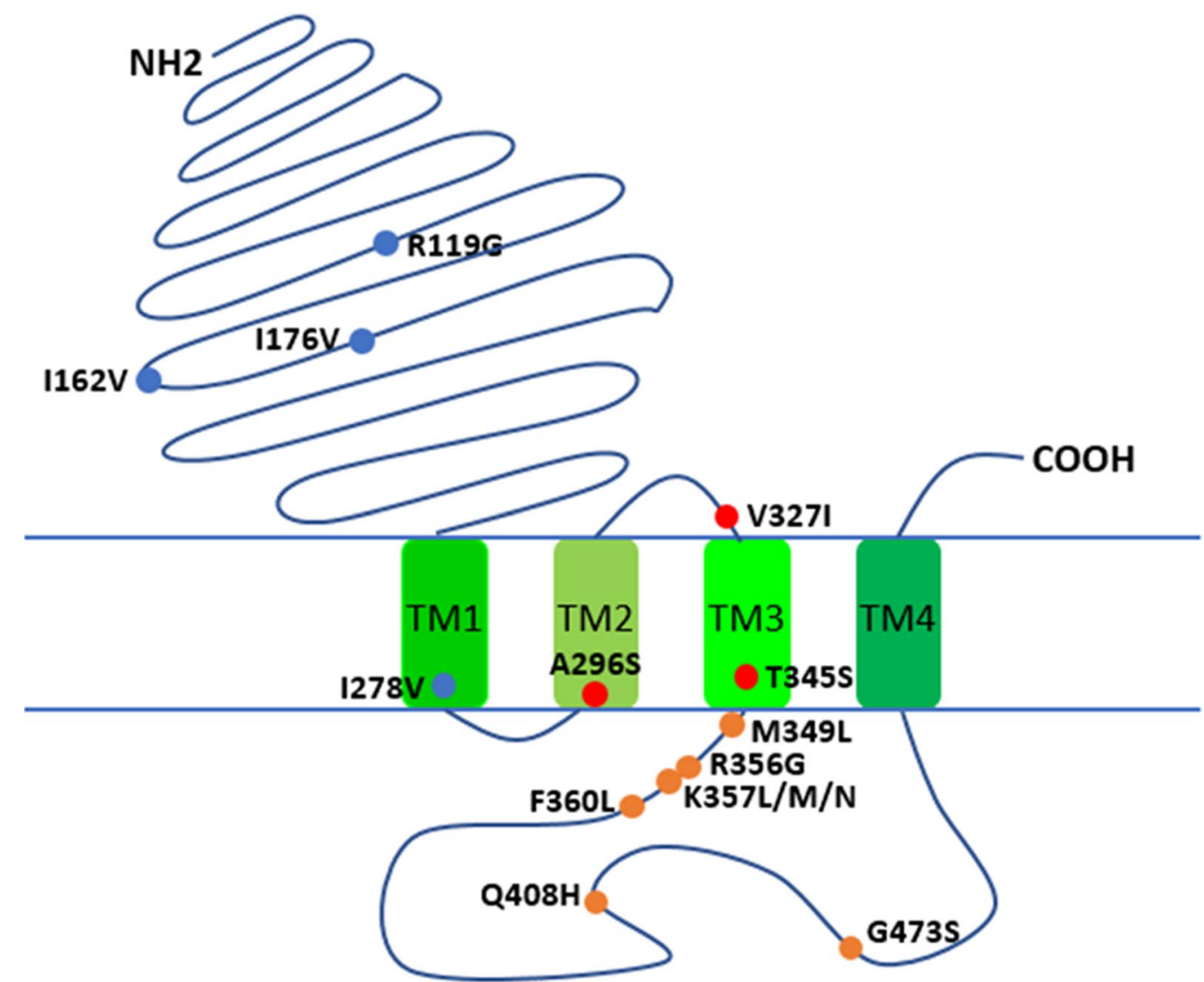

Fig. 1 Schematic representation of polymorphisms in AsRDL monomer. The substitutions indicated by blue dots (R119G, I162V, I176V, I278V) are identified in a laboratory strain. The insecticide resistance-related mutations (296S, 327I, 345S) are marked by red dots. The polymorphisms indicated by orange dots are detected in field mosquitoes (M349L, R356G, K357L/M/N, F360L, Q408H, G473S)

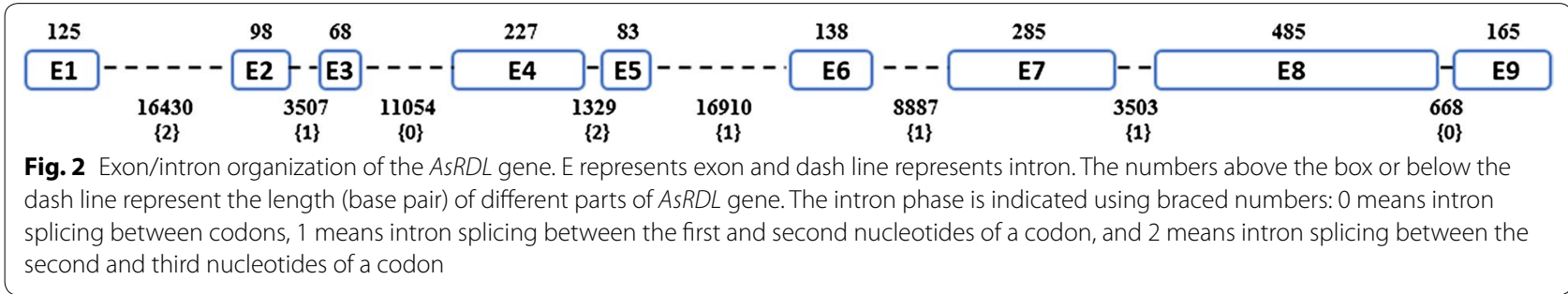

$345 \mathrm{~S}$ was present only in individuals carrying $296 \mathrm{~S}$, and combinations 3, 6 and 7 were distributed in all the nine populations.

The distribution frequencies of the AsRDL genotypes were showed in Fig. 6. Overall, the distribution showed obvious geographical heterogeneity. At position 296, the heterozygotes were present in Baise, Liuzhou and Guilin, while the susceptible homozygotes were found only in Baise and Liuzhou. Notably, the resistant homzygotes (296SS) was fixed in six of the nine populations. In contrast, the frequencies of resistant homozygotes (327II or
345SS) were much lower. Neither individual homozygous for 327II was detected in Hezhou and Wuzhou, nor mosquito homozygous for 345SS was observed in Guigang (Fig. 6).

\section{Haplotype diversity of $A s R D L$ gene}

The sequence data of the $8 \mathrm{R}$ fragment that covers both partial intron 7 and exon 8 with a length of 494 bp were used in haplotype analysis in order to obtain more informative result than data from the 7F fragment. From the sequence data of 240 individuals, 38 AsRDL haplotypes were 
AAA28556 [Drosophila_melanogaster] ASRDL [Anopheles sinensis] AEB60992 [Anopheles_funestus]

AAA28556 [Drosophila_melanogaster] ASRDL [Anopheles sinensis] AEB60992 [Anopheles_funestus]

AAA28556 [Drosophila_melanogaster] ASRDL [Anopheles_sinensis] AEB60992 [Anopheles funestus]

AAA28556 [Drosophila_melanogaster] ASRDL [Anopheles sinensis] AEB60992 [Anopheles_funestus]

AAA28556 [Drosophila melanogaster] ASRDL [Anopheles sinensis] AEB60992 [Anopheles_funestus]

AAA28556 [Drosophila_melanogaster] ASRDL [Anopheles_sinensis] AEB60992 [Anopheles_funestus]

AAA28556 [Drosophila_melanogaster] ASRDL [Anopheles sinensis] AEB60992 [Anopheles_funestus]

AAA28556 [Drosophila melanogaster] ASRDL [Anopheles sinensis] AEB $609 \overline{9} 2$ [Anopheles_funestus]

AAA28556 [Drosophila_melanogaster] ASRDL [Anopheles_sinensis] AEB60992 [Anopheles_funestus]

AAA28556 [Drosophila_melanogaster] ASRDL [Anopheles_sinensis] AEB60992 [Anopheles_funestus]

AAA28556 [Drosophila melanogaster] ASRDL [Anopheles_sinensis] AEB60992 [Anopheles_funestus]
MSDSKMDKLARMAPLPRTP-LLTIWLAINMALIAQETGHKRIHTVQAATGGGSMLGDVNI -----MSLTIEVPHAKSPSLGVLILTLNLALFLPQTINRTPPYVLAGTGGGSMLGDVNI -----MSLTIEVPHAKSPSLGVLILTLNLALFLPQTINRTPPYVLAGTGGGSMLGDVNI

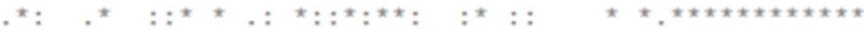

SAILDSFSVSYDKRVRPNYGGPPVEVGVTMYVLSISSVSEVLMDFTLDFYFRQFWTDPRL SAILDSESVGYDKRVRPNYGGPPVEVGVTMYVLSISSLSEVKMDFTLDFYFRQFWTDPRL SAILDSFSVGYDKRVRPNYGGPPVEVGVTMYVLSISSLSEVRMDETLDFYFRQFWTDPRI

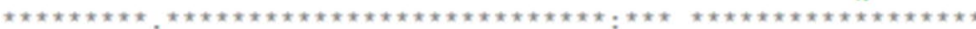

AYRKRPGVETLSVGSEFIKNIWVPDTFFVNEKQSYFHIATTSNEFIRVHHSGSITRSIRL AYRKGPGVETLSVGSEFIKNIWVPDTFFVNEKQSYFHIATTSNEFIRVHHSGSITRSIRI AYRKRPGVETLSVGSEEIKNIWVPDTFFVNEKQSYFHIATTSSEFIRIHHSGSITRSIRL **********************************************************

TITA AEMNQYFPMDRQI IIEIESFGYTMRDIRYFWRDGLSSVGMSSEVELPQERVLG TVTAS DEMGLYFPMDRQI C IIEIESFGYTMRDIRYKWNEGPNSVGVSSEVSLPQFKVLG TITA.SOMGLQYFPMDRQI CIIEIESFGYTMRDIRYKWNEGPNSVGVSSEVSLPQFKVLG

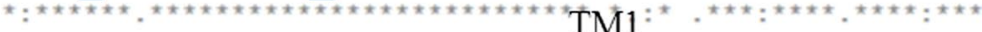

HRQRATEINLTTGNYSRLACEIOEVRSMGYYLIQIYIPSGLIVVISWVSFWLNRNATPAF HRQRAMEISLTTGNYSRLACEIQFVRSMGYYLIQIYIPSGLIVI ISWVSEWLNRNATPAR HRQRAMEISLTT GNYSRLACEIQEVRSMGYYLIQIYIPSGLIVIISWVSFWLNRDATPAF "TM2 $2 * * * * * * * * * * * * * * * * * * * * * * * * * * * * * * * * * * * * * * * * * * *$

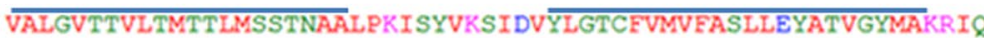
VSLGVTTVLTMTTLMSSTNAALPKISYVKSIDIYLGTCEVMVFASLLEYATVGYMAKRIQ VSLGVTTVLTMTTLMSSTNAALPKISYVKSIDVYLGTCFVMVASLLEYATVGYMAKRIQ ************************************************************ 슬 MRKQRFMAIQKIAEQKKQQLDGANQQQANPNPNANVGGPGGVGVGPGGPGGPGGGVNVGV MRKQRFMAIQKIAEQKKQQAADANHPPPPPP MRKQRFMAIQKIAEQKKQQAADANHPPPPPP

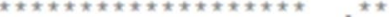

GMGMGPEHGHGHGHHAHSHGHPHAPKOTVSNRPIGFSNICQNVGTRGCSIVGPLEOEVRF ---VASDHSHGHG-HGHSHTHQHTPKQQNGSRSGTMSNVPPNMGSRGCSIVGPLFQEVRF ---VASDHSHGHG-HGHSHTHQHTPKQQMGSRSGTMQNVPPNMGSRGCSIVGPLEQEVRF $\therefore::^{* * * * * *, * * * * *: * * *}: \ldots * \quad: *^{*}: * z^{*}: * * * * * * * * * * * * * *$

KVHDPKAHSKGGTLENTVNGGRGGPQSHGPGPGQGGGPPGGGGGGGGGGGPPEGGGDPEA KVHDPKAHSKGGTLENTINGGRGGGQPGGGGGG----PPGGPPGGGGGGGP-----DEES KVHDPKAHSKGGTLENTINGGRGGGQPGGGGGG----GPPGGPPGGGGGGP-----DEES $* * * * * * * * * * * * * * * * ; * * * * * * * * * * * * *) * * * * * * * * *)$ TM4

AVPAHLLHPGKVKKDINKLLGITPSDIDKYSRIVFPVCFVCFNLMYWIIYLHVSDVVADD AAPQHLIHPG---KDINKLLGITPSDIDKYSRIVFPVCFVCENLMYWI IYLHVSDVVADD AAPQHLIHPG---KDINKLLGITPSDIDKYSRIVEPVCFVCFNLMYWI IYLHVSDVVADD $* * * * ; * * * \quad * * * * * * * * * * * * * * * * * * * * * * * * * * * * * * * * * * * * * * * * * * * * * *$

\section{LVLLGEE- \\ LVLLGEDK \\ LVLLGEEK}

$\approx * \cdots * *$

Fig. 3 Alignment of amino acid sequences of RDLs from Anopheles sinensis (AsRDL), An. funestus (AEB60992) and Drosophila melanogaster (AAA28556). The asterisks $(*)$ indicate identical amino acid, colons (:) represent conserved substitution and black dots (.) indicate weakly conserved sites. The three insecticide resistance-related residues $(296,327,345)$ are marked with arrows. The four transmembrane regions (TM1-4) are indicated by a straight line. The two cysteines forming the cys-loop are in box

identified (Table 3). The 38 haplotypes varied in frequency from 0.2 to $34.8 \%$. Overall five common haplotypes $(\mathrm{H} 1$, $\mathrm{H} 2, \mathrm{H} 3, \mathrm{H} 17$ and H25) accounted for $67 \%$ of the haplotype diversity, while sixteen haplotypes were observed only once. The two most common haplotypes (H3 and H17) occurred in all the nine populations.
The combination of insecticide resistance-related mutation at position 345 (i.e. 345S) with the other polymorphic sites resulted in 9 resistant haplotypes $(\mathrm{H} 2$, H17-22, H32-33) with frequency ranging from 0.2 to $13.1 \%$ (Table 3). The nine resistant haplotypes accounted for $36 \%$ of the haplotype diversity. Four of them $(\mathrm{H} 2, \mathrm{H} 17$, 


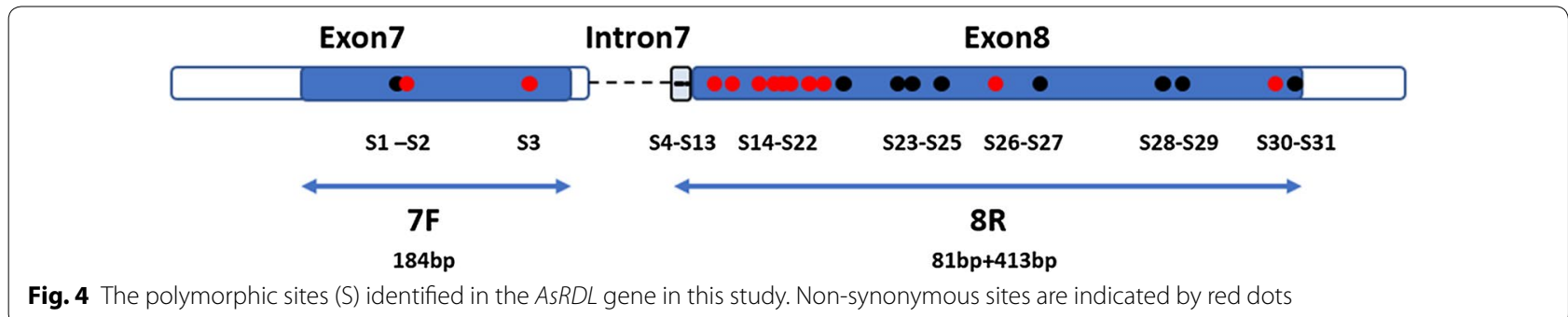

Fig. 4 The polymorphic sites (S) identified in the AsRDL gene in this study. Non-synonymous sites are indicated by red dots

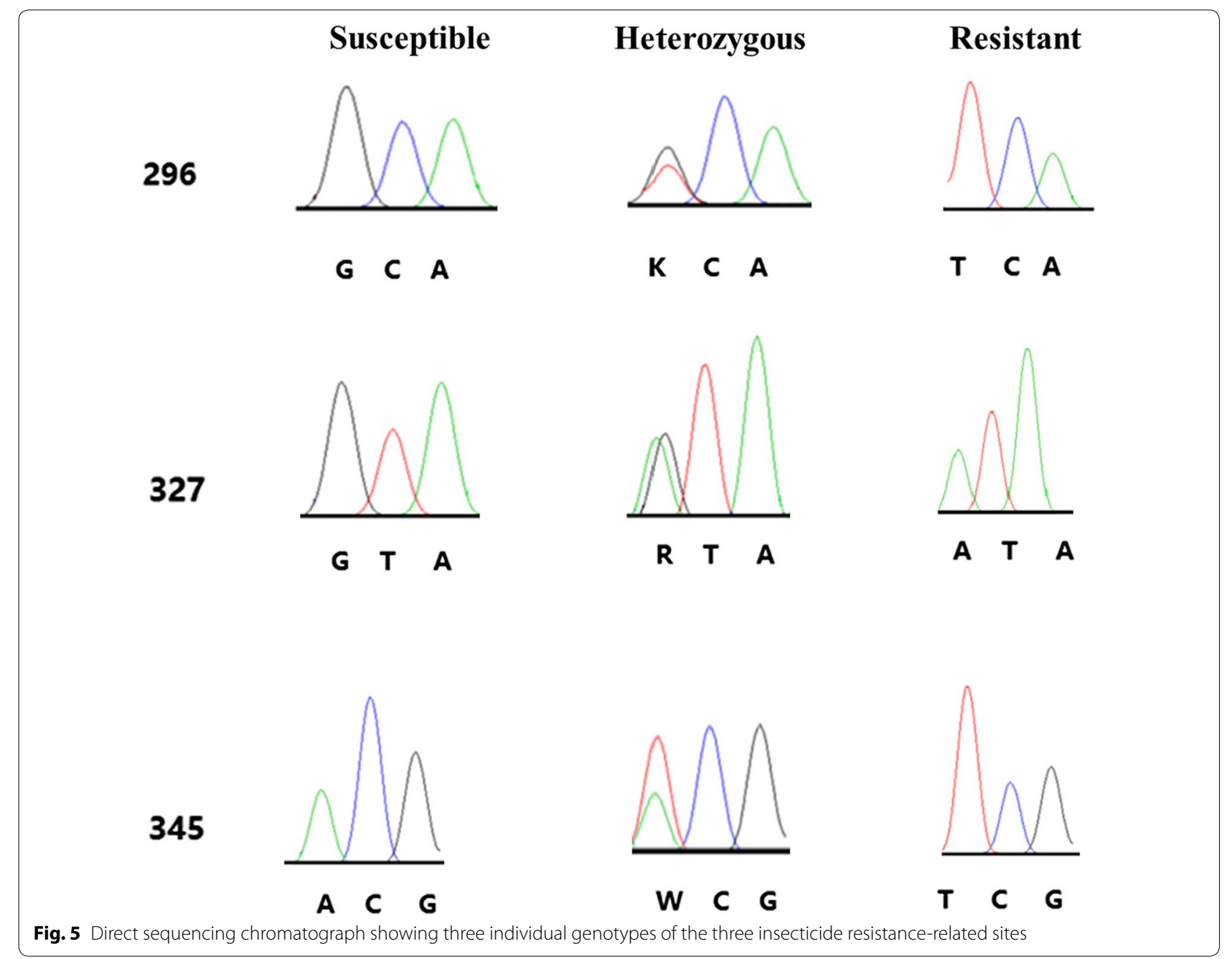

$\mathrm{H} 18$ and $\mathrm{H} 21$ ) were commonly detected (frequency $>5 \%$ ) and distributed in most of nine populations. The number of resistant haplotypes in each of the nine populations ranged from 3 (Hechi) to 6 (Yulin and Wuzhou).

\section{Genealogical analysis of $A s R D L$ haplotypes}

The network analysis revealed a complex reticulate genealogical relation of the 38 AsRDL haplotypes, and suggested independent mutation events leading 


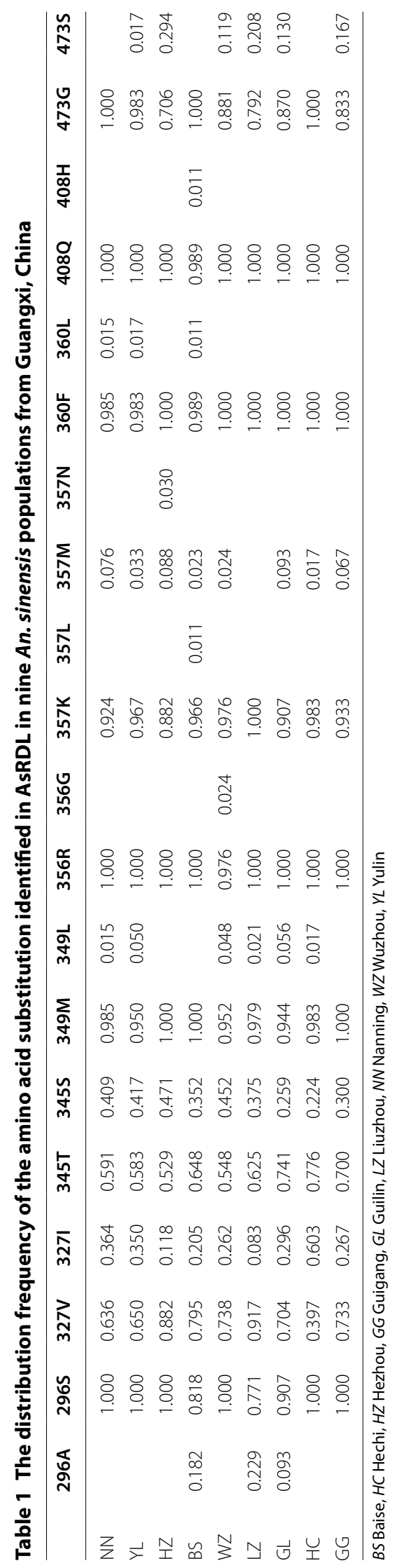




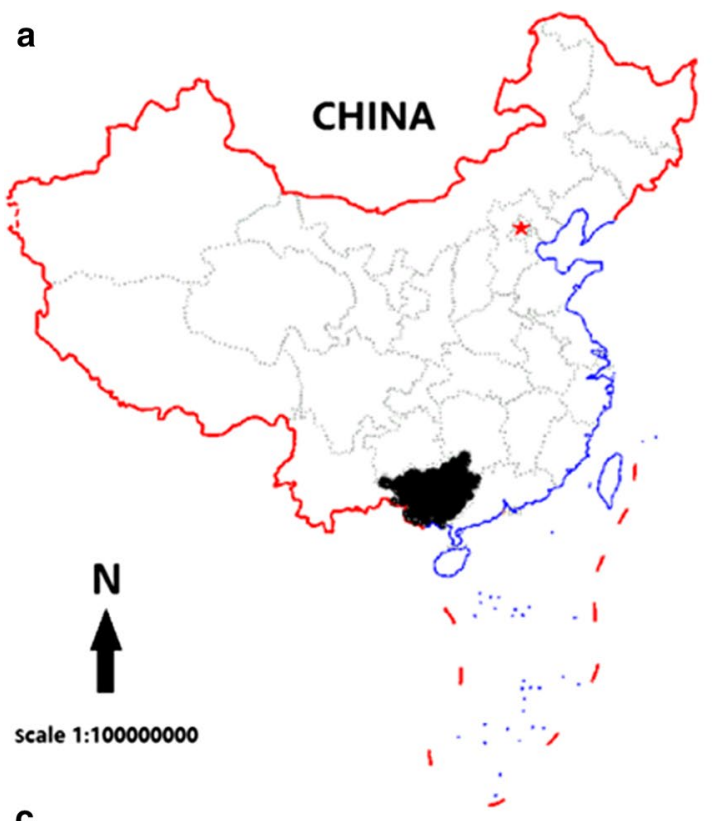

b
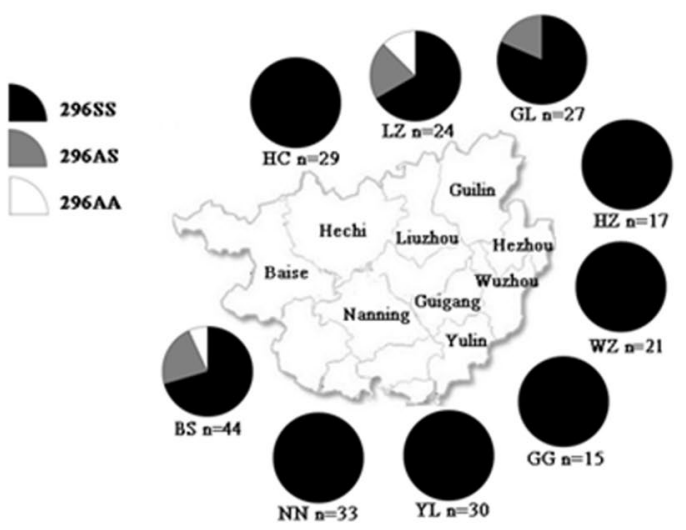

C

d
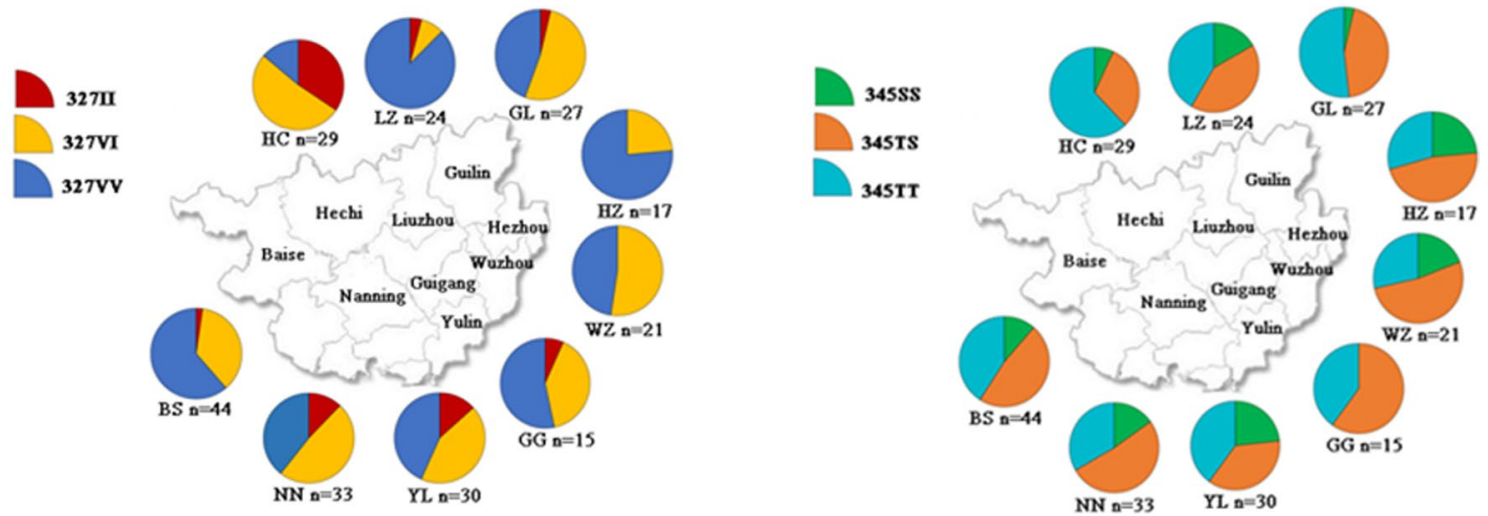

Fig. 6 The sampling location (a), and distribution frequencies of individual genotypes of AsRDL in Guangxi (b-d). BS Baise, HC Hechi, HZ Hezhou, GG Guigang, GL Guilin, LZ Liuzhou, NN Nanning, WZ Wuzhou, YL Yulin. $n$ represents the sample size

to 345S-containing haplotypes (Fig. 7). For example, the resistant $\mathrm{H} 17$ was possibly derived from $\mathrm{H} 3$, while $\mathrm{H} 32$ from $\mathrm{H} 25$. Although the phylogenetic tree had lower resolution, the 38 haplotypes were roughly grouped into two major clades (63\% bootstrap value) separated by 3 nucleotide substitutions in intron 7 (Fig. 8).

\section{Discussion}

In insects, GABA receptors are ligand-gated chloride channels consisting of presumably the homopentameric RDL subunit encoded by the $R D L$ gene. RDLs mediate synapse inhibition in the central nervous system and are important targets for insecticides of widely varied structures such as cyclodienes and fipronil [14]. In this study, 
Table 2 Frequency (in percentage) of combinations of insecticide resistance-associated sites in AsRDL

\begin{tabular}{|c|c|c|c|c|c|c|c|c|c|c|c|c|}
\hline & \multicolumn{3}{|c|}{ Residue sites } & \multicolumn{9}{|c|}{ Populations } \\
\hline & 296 & 327 & 345 & NN & YL & $\mathrm{HZ}$ & BS & WZ & $\mathrm{LZ}$ & $\mathrm{GL}$ & $\mathrm{HC}$ & GG \\
\hline Combination 1 & $S / S$ & I/I & $\mathrm{T} / \mathrm{T}$ & 12.1 & 13.3 & & 2.3 & & 4.2 & 3.7 & 34.5 & 6.7 \\
\hline Combination 2 & $S / S$ & IN & $S / S$ & & & & 2.3 & & & & 3.4 & \\
\hline Combination 3 & $S / S$ & IN & $\mathrm{T} / \mathrm{S}$ & 36.4 & 23.3 & 23.5 & 11.4 & 33.3 & 4.2 & 22.2 & 24.1 & 26.7 \\
\hline Combination 4 & $S / S$ & IN & $\mathrm{T} / \mathrm{T}$ & 12.1 & 20.0 & & 18.2 & 19.0 & & 25.9 & 24.1 & 13.3 \\
\hline Combination 5 & $S / S$ & VN & $S / S$ & 15.2 & 23.3 & 23.5 & 9.1 & 19.0 & 16.7 & 3.7 & 3.4 & \\
\hline Combination 6 & $S / S$ & VN & $\mathrm{T} / \mathrm{S}$ & 15.2 & 13.3 & 23.5 & 25.0 & 19.0 & 29.2 & 18.5 & 6.9 & 33.3 \\
\hline Combination 7 & $S / S$ & VN & $\mathrm{T} / \mathrm{T}$ & 9.1 & 6.7 & 29.4 & 2.3 & 9.5 & 12.5 & 7.4 & 3.4 & 20.0 \\
\hline Combination 8 & $S / A$ & IN & $\mathrm{T} / \mathrm{T}$ & & & & 4.5 & & 4.2 & 3.7 & & \\
\hline Combination 9 & $S / A$ & VN & $\mathrm{T} / \mathrm{S}$ & & & & 11.4 & & 8.3 & 3.7 & & \\
\hline Combination 10 & $S / A$ & VN & $\mathrm{T} / \mathrm{T}$ & & & & 6.8 & & 8.3 & 11.1 & & \\
\hline Combination 11 & $\mathrm{~A} / \mathrm{A}$ & VN & $\mathrm{T} / \mathrm{T}$ & & & & 6.8 & & 12.5 & & & \\
\hline
\end{tabular}

The putatively resistant mutations are in italic. The blank cells indicate 0 values

BS Baise, HC Hechi, HZ Hezhou, GG Guigang, GL Guilin, LZ Liuzhou, NN Nanning, WZ Wuzhou, YL Yulin

the $R D L$ gene was identified in $A n$. sinensis. The putative AsRDL protein has 557 amino acid residues and shares the common structural features with known Cys-loop ligand-gated chloride channels (Fig. 1). Similar to the finding obtained in An. funestus [18], only one GABAreceptor isoform is found in An. sinensis in this study.

The RDL subunit has four transmembrane segments (M1, M2, M3 and M4). Point mutations in the M2 and M3 have been documented to confer low sensitivity of the binding site and thus insecticide resistance. In this study, the insecticide resistance associated mutation 296S, and two other mutations (327I and 345S) that have been documented to be linked to $296 \mathrm{~S}$ [18, 23], were identified and widely distributed in An. sinensis populations (Table 1 and Fig. 5). The prediction of the three mutations to be resistance-related is made by reference to other species, thus further functional study is required to confirm it. Whether other mutations (356G, 357L, 357N and $408 \mathrm{H})$ in An. sinensis, which are narrowly distributed at a low frequency, contribute to insecticide resistance is unclear.

Point mutations (Ala to Ser/Gly/Asn) in RDL homologues at the site equivalent to 301 in Drosophila have been documented to provide resistance to cyclodienes and phenylpyrazoles in several species [17-24, 34]. From the 240 individuals from nine geographical locations, the $296 \mathrm{~S}$ mutation was detected at a very high frequency. Notably, $100 \%$ of individuals in six of the nine populations carried this mutation in the homozygous form. These data indicate a risk of insecticide to cyclodienes and phenylpyrazoles in Guangxi An. sinensis populations. However, the GCT to GGT mutation (inducing the A296G change) found in An. gambiae [17], and the replacement of Ala with Asn observed in plant hoppers [20, 21], were not detected in An. sinensis samples. Given that cyclodienes have now been withdrawn from control programmes, the high frequency of the dieldrin resistance-associated A296S mutation in An. sinensis in Guangxi may be the result of the increasing use of insecticides targeting the GABA receptor (e.g. ethiprole) in the agriculture [35].

The 327I mutation which is located in the intracellular loop between TM2 and TM3 has been reported to coexist with $296 \mathrm{~S}$ in dieldrin-resistant An. funestus [18]. Interestingly, a point mutation identical to An. funestus (GTA to ATA) was identified in An. sinensis in this study (Fig. 5). The impact of the 327I mutation is unknown and worthy of further investigation.

Two mutations at the site 350 in the third membrane spanning domain of RDL have been documented in Drosophila [22, 23]. T350 M was observed in fipronil resistant Drosophila simulans with Gly301 [22], while T350S was detected in a Drosophila melanogaster line (Ral-491) that is homozygous for Ser301 [23]. Interestingly, conserved mutations (A296G + T345 M) were also found in An. gambiae [13]. In this study, parallel 
Table 3 Haplotypes of the AsRDL gene and their frequencies in nine An. sinensis populations from Guangxi, China

\begin{tabular}{|c|c|c|c|c|c|c|c|c|c|c|c|c|c|}
\hline Haplotype & Polymorphic sites & Intron type & 345aa & NN & $\mathrm{YL}$ & $\mathrm{HZ}$ & BS & WZ & LZ & GL & $\mathrm{HC}$ & GG & Total numbers \\
\hline $\mathrm{H} 1$ & ACCCTGATTGAACAAGTCGGCCGGGGGT & [1] & $\mathrm{T}$ & 4 & 2 & 1 & 4 & 3 & 4 & 3 & 6 & 2 & 29 \\
\hline $\mathrm{H} 2$ & ACCCTGATTGTACAAGTCGGCCGGGGGT & {$[1]$} & S & 7 & 9 & & 7 & 2 & & & 3 & 1 & 29 \\
\hline $\mathrm{H} 3$ & ACCCTGCTTGAACAAGTCGGCCGGGGGT & {$[2]$} & $\mathrm{T}$ & 26 & 28 & 9 & 22 & 13 & 10 & 21 & 28 & 10 & 167 \\
\hline $\mathrm{H} 4$ & ACCCTGCTTGAACAAGTCGGCCGGGGGC & {$[2]$} & $\mathrm{T}$ & & & & 1 & & & & & & 1 \\
\hline $\mathrm{H} 5$ & ACCCTGCTTGAACAAGTCGGCCGGGGAT & {$[2]$} & $\mathrm{T}$ & & & 3 & & 1 & 2 & 3 & & 2 & 11 \\
\hline H6 & ACCCTGCTTGAACAAGTCGGCCGGATGC & {$[2]$} & $\mathrm{T}$ & & & & & & & 1 & & & 1 \\
\hline $\mathrm{H} 7$ & ACCCTGCTTGAACAAGTCGGCCCGATGC & {$[2]$} & $\mathrm{T}$ & & & & 1 & & & & & & 1 \\
\hline $\mathrm{H} 8$ & ACCCTGCTTGAACAAGTCGGTCGGGGGT & {$[2]$} & $\mathrm{T}$ & & & & 1 & & & & & & 1 \\
\hline H9 & ACCCTGCTTGAACAAGTCGACCGGGGGT & {$[2]$} & $\mathrm{T}$ & & & & & & 1 & & & & 1 \\
\hline $\mathrm{H} 10$ & ACCCTGCTTGAACAAGTGGGCCGGGGGT & [2] & $\mathrm{T}$ & & 1 & & & & & & & & 1 \\
\hline $\mathrm{H} 11$ & ACCCTGCTTGAACAAGCCGGCCGGGGGT & [2] & T & 1 & & & 1 & & & 1 & 2 & & 5 \\
\hline $\mathrm{H} 12$ & ACCCTGCTTGAACAACTCGGCCGGGGGT & {$[2]$} & $\mathrm{T}$ & & & 1 & & & & & & & 1 \\
\hline $\mathrm{H} 13$ & ACCCTGCTTGAACATGTCGGCCGGGGGT & {$[2]$} & $\mathrm{T}$ & 5 & 2 & & & & & 1 & 1 & 1 & 10 \\
\hline $\mathrm{H} 14$ & ACCCTGCTTGAACTTGTCGGCCGGGGGT & {$[2]$} & $\mathrm{T}$ & & & & 1 & & & & & & 1 \\
\hline $\mathrm{H} 15$ & ACCCTGCTTGAAGAAGTCGGCCGGGGGT & {$[2]$} & $\mathrm{T}$ & & & & & 1 & & & & & 1 \\
\hline $\mathrm{H} 16$ & ACCCTGCTTGATCAAGTCGGCCGGGGGT & {$[2]$} & $\mathrm{T}$ & 1 & & & 1 & 1 & 1 & & & & 4 \\
\hline H17 & ACCCTGCTTGTACAAGTCGGCCGGGGGT & {$[2]$} & S & 13 & 12 & 2 & 17 & 8 & 2 & 2 & 4 & 3 & 63 \\
\hline H18 & ACCCTGCTTGTACAAGTCGGCCGGGGAT & {$[2]$} & S & & 1 & 7 & & 4 & 8 & 4 & & 3 & 27 \\
\hline H19 & ACCCTGCTTGTACAAGTCGGCCGGGTGC & {$[2]$} & S & 1 & & & & & & & & & 1 \\
\hline $\mathrm{H} 2 \mathrm{O}$ & ACCCTGCTTGTACAAGTCGGCCGGATGT & {$[2]$} & S & & & & 1 & & & 1 & & & 2 \\
\hline$H 21$ & ACCCTGCTTGTACAAGTCGGCCGGATGC & {$[2]$} & $\mathrm{S}$ & 6 & 1 & 1 & 5 & 1 & 1 & 4 & 6 & & 25 \\
\hline $\mathrm{H} 22$ & ACCCTGCTTGTTCAAGTCGGCCGGGGGT & {$[2]$} & S & & 1 & & 1 & & & & & & 2 \\
\hline $\mathrm{H} 23$ & ACCCTGCTTAAACAAGTCGGCCGGGGGT & {$[3]$} & $\mathrm{T}$ & & & 1 & & & 2 & 1 & & 4 & 8 \\
\hline $\mathrm{H} 24$ & ACCCTGCTTAAACATGTCGGCCGGGGGT & {$[3]$} & $\mathrm{T}$ & & & & & & & 1 & & 1 & 2 \\
\hline $\mathrm{H} 25$ & ACCCCGCACGAACAAGTCGGCCGGGGGT & {$[4]$} & $\mathrm{T}$ & 2 & & & 18 & & 5 & 1 & 7 & & 33 \\
\hline $\mathrm{H} 26$ & ACCCCGCACGAACAAGTCGGCCGGAGGT & {$[4]$} & $\mathrm{T}$ & & & & & & 1 & & & & 1 \\
\hline $\mathrm{H} 27$ & ACCCCGCACGAACAAGTCGGCTGGGGGT & {$[4]$} & $\mathrm{T}$ & & & & & & & 1 & & & 1 \\
\hline $\mathrm{H} 28$ & ACCCCGCACGAACAAGTCAGCCGGGGGT & {$[4]$} & $\mathrm{T}$ & & & & & 1 & & & & & 1 \\
\hline $\mathrm{H} 29$ & ACCCCGCACGAACATGTCGGCCGGGGGT & {$[4]$} & $\mathrm{T}$ & & & 3 & 1 & 1 & & 2 & & & 7 \\
\hline $\mathrm{H} 30$ & ACCCCGCACGAACATGTCGGCCGAGGGT & {$[4]$} & $\mathrm{T}$ & & & & 1 & & & 1 & & & 2 \\
\hline $\mathrm{H} 31$ & ACCCCGCACGATCAAGTCGGCCGGGGGT & {$[4]$} & $\mathrm{T}$ & & 2 & & & 1 & & 3 & 1 & & 7 \\
\hline H32 & ACCCCGCACGTACAAGTCGGCCGGGGGT & {$[4]$} & S & & 1 & & & 2 & & & & & 3 \\
\hline H33 & ACCCCGCACGTACAAGTCGGCTGGGGGT & {$[4]$} & S & & & 6 & & 2 & 7 & 3 & & 2 & 20 \\
\hline $\mathrm{H} 34$ & ACCTTGCTTGAACAAGTCGGCCGGGGGT & {$[5]$} & $\mathrm{T}$ & & & & 2 & 1 & 3 & & & & 6 \\
\hline $\mathrm{H} 35$ & ACCTTGCTTGAACAAGTCGGCCGAGGGT & {$[5]$} & $\mathrm{T}$ & & & & 2 & & & & & & 2 \\
\hline $\mathrm{H} 36$ & ACCTTCCTTGAACAAGTCGGCCGGGGGT & {$[6]$} & $\mathrm{T}$ & & & & & & 1 & & & & 1 \\
\hline $\mathrm{H} 37$ & ACACCGCACGAACAAGTCGGCCGGGGGT & {$[7]$} & $\mathrm{T}$ & & & & & & & & & 1 & 1 \\
\hline $\mathrm{H} 38$ & TTCCCGCACGAACAAGTCGGCCGGGGGT & {$[8]$} & $\mathrm{T}$ & & & & 1 & & & & & & 1 \\
\hline $2 \mathrm{~N}$ & & & & 66 & 60 & 34 & 88 & 42 & 48 & 54 & 58 & 30 & 480 \\
\hline
\end{tabular}

The haplotypes containing the $345 \mathrm{~S}$ mutation are in italics

BS Baise, HC Hechi, HZ Hezhou, GG Guigang, GL Guilin, LZ Liuzhou, NN Nanning, WZ Wuzhou, YL Yulin 


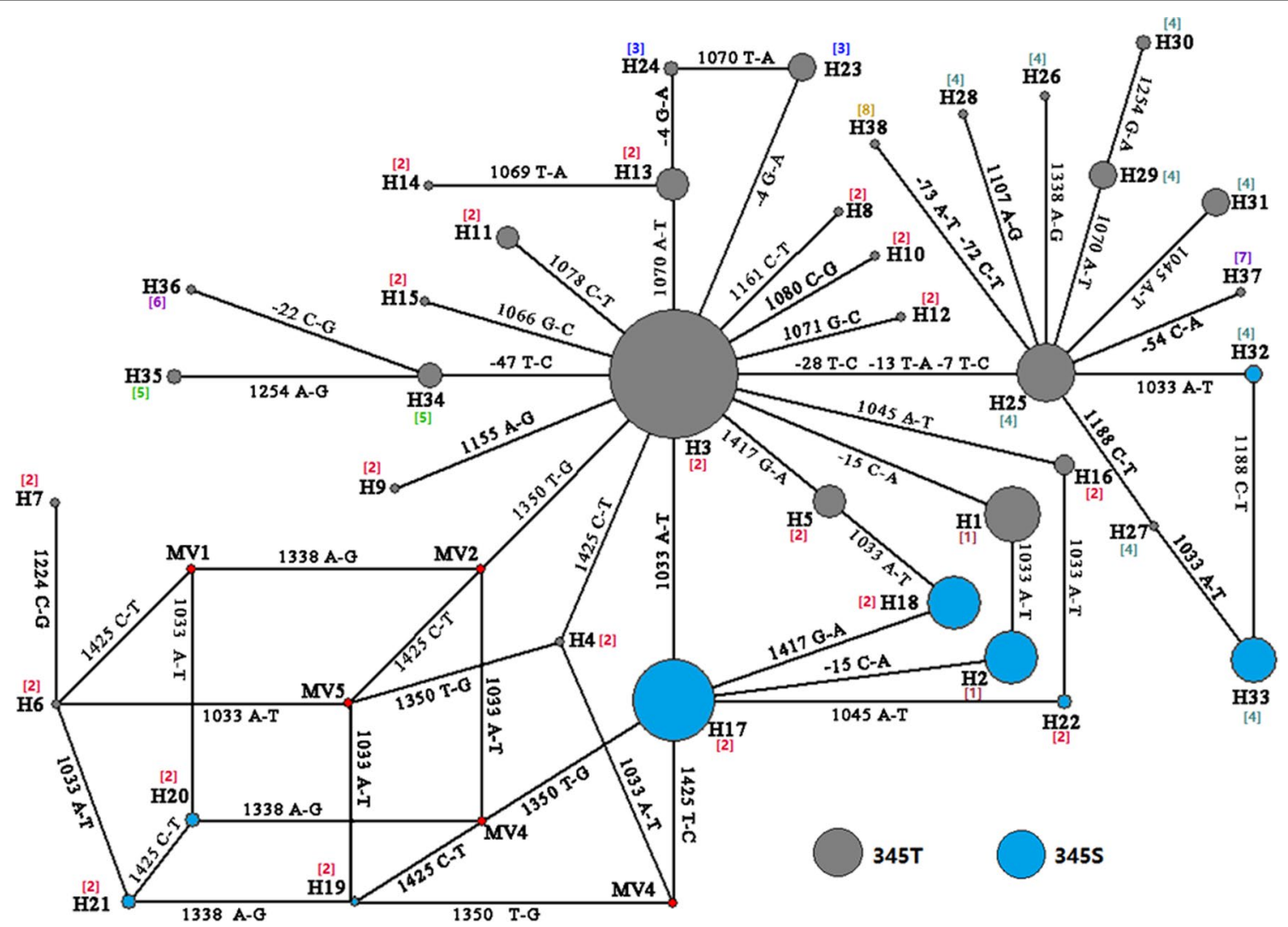

Fig. 7 Network showing the genealogy of $A s R D L$ haplotypes. The size of each circle is proportional to the corresponding frequency of a certain haplotype identified in Guangxi. Straight line indicates the possible mutational step. The note near the connecting line is referred to the mutation position and base. The intron type for each haplotype is indicated by corresponding number in bracket

double mutations (A296S + T345S, corresponding to A301S + T350S/M in Drosophila) were detected in An. sinensis. This observation lends support to the notion that there is a functional role for mutations at the 345 site $[13,23]$.

Sequence analyses revealed that $A s R D L$ gene had diverse polymorphisms. From the fragment sequences of AsRDL from 240 mosquitoes, 38 haplotypes were identified (Table 3). The number and frequency of $A s R D L$ haplotypes were different among the nine populations from Guangxi. The most abundant haplotype was H3 (34.8\%), followed by H17 (13\%). Both H3 and $\mathrm{H} 17$ were distributed in all the nine populations tested, and shared the type-2 intron. Similar to the status of $A S-V G S C$ [12], the high polymorphism of $A s R D L$ may be partly explained by their large population size, wide distribution range of An. sinensis [36], diverse natural landscapes and different local insecticide selective pressure in Guangxi.

The genealogical relation and phylogenetic tree analyses support two independent origins of the resistance related 345S mutation in AsRDL (Figs. 7, 8). The two most common susceptible haplotypes (H3 and H25) could be considered as possible ancestors of resistant haplotypes respectively. The six resistant haplotypes (H17-22) were derived from their common susceptible ancestor $\mathrm{H} 3$ with one to four mutational steps, whereas the other two resistant haplotypes (H32 and H33) were the result of one and two mutational steps respectively from $\mathrm{H} 25$. 


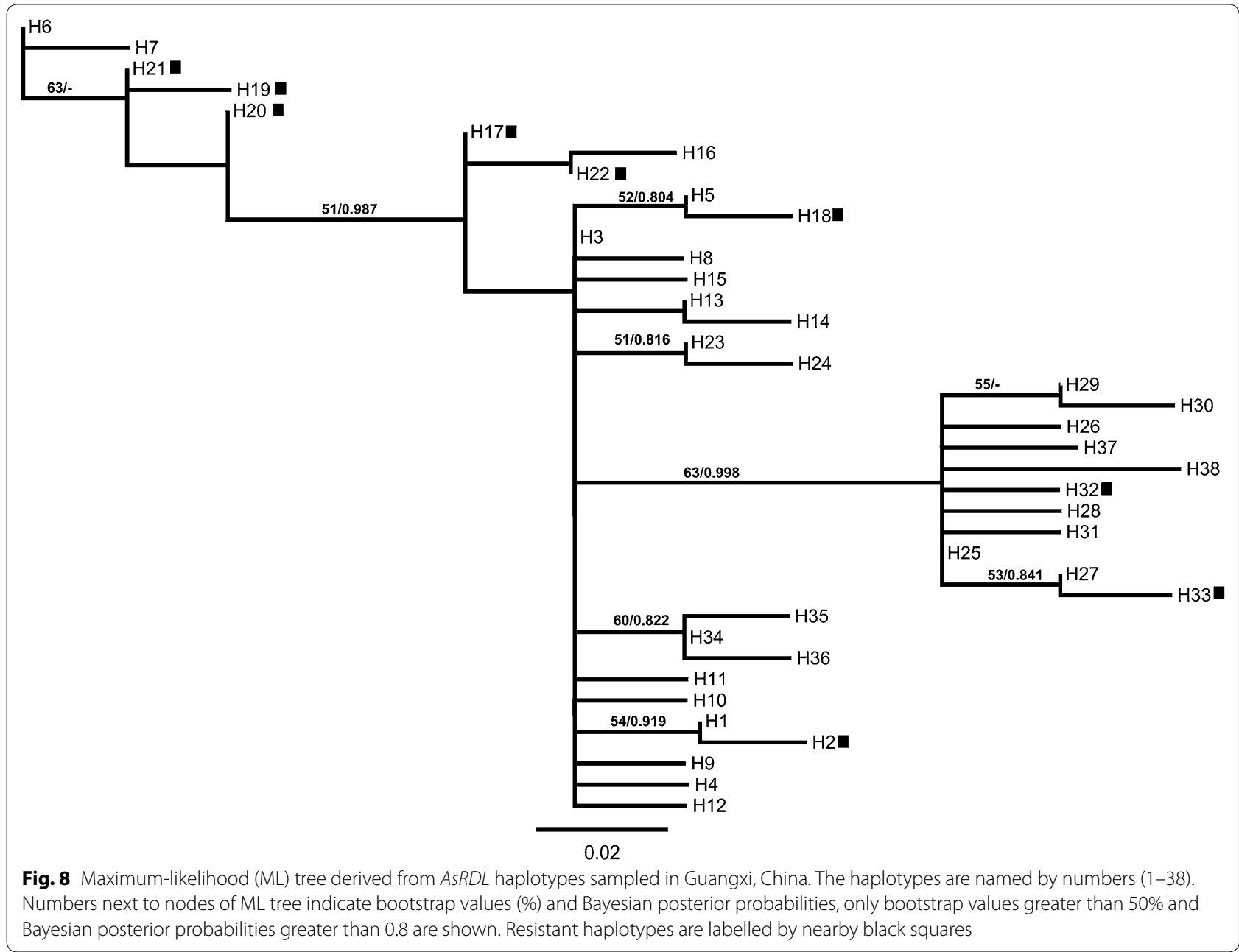

\section{Conclusions}

Three insecticide resistance related mutations (296S, 327I and 345S) in AsRDL were detected in Guangxi. The extremely high frequency or fixation of the $296 \mathrm{~S}$ mutation, and the occurrence of $327 \mathrm{I}$ and $345 \mathrm{~S}$ in addition to 296S, in all populations tested strongly indicate a risk of multiple and/or cross resistance to cyclodienes (e.g. dieldrin and $\alpha$-endosulfan) and phenylpyrazole (e.g. fipronil and ethiprole). The haplotype diversity plus genetic heterogeneities in the geographical distribution, and multiple origins of $A s R D L$ alleles call for a location-customized strategy for monitoring and management of insecticide resistance.

\section{Abbreviations}

RDL: resistant to dieldrin; Guangxi: Guangxi Zhuang Autonomous Region; GABA: gamma-aminobutyric acid.

\section{Authors' contributions}

$\mathrm{XHQ}$ and $\mathrm{XYF}$ conceived the study. CY performed the experiment. $\mathrm{CY}, \mathrm{ZSH}$ and $\mathrm{XHQ}$ analysed the data. XHQ, CY and ZSH wrote the paper. XYF and ML contributed to sample collection. All authors read and approved the final manuscript.

\section{Author details}

1 State Key Laboratory of Integrated Management of Pest Insects and Rodents, Institute of Zoology, Chinese Academy of Sciences, Beijing 100101, China. ${ }^{2}$ University of Chinese Academy of Sciences, Beijing 100049, China. ${ }^{3}$ Guangxi Zhuang Autonomous Region Centre for Diseases Control and Prevention, Nanning 530028, China.

\section{Acknowledgements}

The authors thank the reviewers for their constructive comments and suggestions.

\section{Competing interests}

The authors declare that they have no competing interests.

Availability of data and materials

All the datasets are presented in the main paper.

Consent for publication

Not applicable.

Ethics approval and consent to participate

Not applicable. 


\section{Funding}

This work was supported by $2017 Z X 10303404002004$. The funders had no role in the study design, data collection, analysis, decision to publish or preparation of the manuscript.

\section{Publisher's Note}

Springer Nature remains neutral with regard to jurisdictional claims in published maps and institutional affiliations.

Received: 18 October 2017 Accepted: 23 November 2017

Published online: 28 November 2017

\section{References}

1. Li JH, Wei SJ, Yang YC, Li J. Analysis of the prevalence of imported malaria in Guangxi from 2012 to 2013. J Pathog Biol. 2015;10:180-3.

2. Li JH, Li J, Qin YX, Guo CK, Huang YM, Lin Z, et al. Appraisal of the effect and measures on control malaria for sixty years in Guangxi. J Trop Med. 2014:14:361-4

3. Li J, Yang YC, Zhang WW, Wei SJ, Lin KM. Mid-term assessment report of malaria elimination action plan in Guangxi. China Trop Med. 2016;16:305-10

4. Lu GY, Zhou SS, Horstick O, Wang X, Liu YL, Müller O. Malaria outbreaks in China (1990-2013): a systematic review. Malar J. 2014;13:269.

5. Lin KM, Huang YM, Li J, Mao W, Li JH, Qin YX, et al. Malaria vector Anopheles investigation analysis after malaria was basically eliminated in Guangxi. J Med Pest Control. 2014;30:963-6.

6. Guo CK, Li JH, Qin YX. Investigation on geographical distribution, ecological feature and malaria transmission of Anopheles in Guangxi. Chin J Vector Biol Control. 2007:18:112-5.

7. Enayati A, Hemingway J. Malaria management: past, present, and future. Annu Rev Entomol. 2010:55:569-91.

8. Lu B. Integrated mosquito management. 2nd ed. Beijing: Science Press; 1999

9. Wang DQ, Xia ZG, Zhou SS, Zhou XN, Wang RB, Zhang QF. A potential threat to malaria elimination: extensive deltamethrin and DDT resistance to Anopheles sinensis from the malaria-endemic areas in China. Malar J. 2013;12:164.

10. Zhang HW, Liu Y, Hu T, Zhou RM, Chen JS, Qian D, et al. Knockdown resistance of Anopheles sinensis in Henan province, China. Malar J. 2015;14:137.

11. Feng $X Y$, Yang C, Yang YC, Li J, Lin KM, Li M, et al. Distribution and frequency of G119S mutation in ace-1 gene within Anopheles sinensis populations from Guangxi, China. Malar J. 2015;14:470.

12. Yang C, Feng XY, Huang ZS, Li M, Qiu XH. Diversity and frequency of $k d r$ mutations within Anopheles sinensis populations from Guangxi, China. Malar J. 2016;15:411.

13. Taylor-Wells J, Brooke BD, Bermudez I, Jones AK. The neonicotinoid imidacloprid, and the pyrethroid deltamethrin, are antagonists of the insect Rdl GABA receptor. J Neurochem. 2015;135:705-13.

14. Casida JE, Durkin KA. Novel GABA receptor pesticide targets. Pestic Biochem Physiol. 2015;121:22-30.

15. ffrench-Constant RH, Williamson MS, Emyr Davies TG, Bass C. Ion channels as insecticide targets. J Neurogenet. 2016;30:163-77.

16. ffrench-Constant $\mathrm{RH}$, Anthony N, Aronstein K, Rocheleau T, Stilwell G. Cyclodiene insecticide resistance: from molecular to population genetics. Annu Rev Entomol. 2000:48:449-66.

17. Du W, Awolola TS, Howell P, Koekemoer LL, Brooke BD, Benedict MQ, et al. Independent mutations in the Rdl locus confer dieldrin resistance to Anopheles gambiae and Anopheles arabiensis. Insect Mol Biol. 2005;14:179-83.
18. Wondji CS, Dabire RK, Tukur Z, Irving H, Djouaka R, Morgan JC. Identification and distribution of a GABA receptor mutation conferring dieldrin resistance in the malaria vector Anopheles funestus in Africa. Insect Biochem Mol Biol. 2011;41:484-91.

19. Tantely ML, Tortosa P, Alout H, Berticat C, Berthomieu A, Rutee A, et al. Insecticide resistance in Culex pipiens quinquefasciatus and Aedes albopictus mosquitoes from La Réunion Island. Insect Biochem Mol Biol. 2010;40:317-24.

20. Nakao T, Kawase A, Kinoshita A, Abe R, Hama M, Kawahara N, et al. The $A 2^{\prime} \mathrm{N}$ mutation of the RDL $\gamma$-aminobutyric acid receptor conferring fipronil resistance in Laodelphax striatellus (Hemiptera: Delphacidae). J Econ Entomol. 2011;104:646-52.

21. Wei Q, Wu SF, Gao CF. Molecular characterization and expression pattern of three GABA receptor-like subunits in the small brown planthopper Laodelphax striatellus (Hemiptera: Delphacidae). Pestic Biochem Physiol. 2017:136:34-40.

22. Le Goff G, Hamon A, Berge JB, Amichot M. Resistance to fipronil in Drosophila simulans: influence of two point mutations in the RDL GABA receptor subunit. J Neurochem. 2005;92:1295-305.

23. Remnant EJ, Morton CJ, Daborn PJ, Lumb C, Yang YT, Ng HL, et al. The role of Rdl in resistance to phenylpyrazoles in Drosophila melanogaster. Insect Biochem Mol Biol. 2014:54:11-21.

24. Zhang YX, Meng XK, Yang YX, Li H, Wang X, Yang BJ, et al. Synergistic and compensatory effects of two point mutations conferring targetsite resistance to fipronil in the insect GABA receptor RDL. Sci Rep. 2016:6:32335

25. Lu B, Wu H. Classification and identification of important medical insects of China. 2nd ed. Zhengzhou: Henan Science and Technology Publishing House; 2003.

26. Joshi D, Park MH, Saeung A, Choochote W, Min GS. Multiplex assay to identify Korean vectors of malaria. Mol Ecol Resour. 2010;10:748-50.

27. Tan WL, Wang ZM, Li CX, Chu HL, Xu Y, Dong DY, et al. First report on co-occurrence knockdown resistance mutations and susceptibility to beta- cypermethrin in Anopheles sinensis from Jiangsu Province, China. PLOS ONE. 2012;7:e29242-341.

28. Rinkevich FD, Zhang L, Hamm RL, Brady SG, Lazzaro BP, Scott JG. Frequencies of the pyrethroid resistance alleles of Vssc1 and CYP6D1 in house flies from the eastern United States. Insect Mol Biol. 2006:15:157-67.

29. Stephens M, Scheet P. Accounting for decay of linkage disequilibrium in haplotype inference and missing-data imputation. Am J Hum Genet. 2005;76:449-62.

30. Darriba D, Taboada GL, Doallo R, Posada D. jModelTest 2: more models, new heuristics and parallel computing. Nat Methods. 2012;9:772.

31. Guindon S, Dufayard JF, Lefort V, Anisimova M, Hordijk W, Gascuel O. New algorithms and methods to estimate maximum-likelihood phylogenies: assessing the performance of PhyML 3.0. Syst Biol. 2010;59:307-21.

32. Huelsenbeck JP, Ronquist F. MRBAYES: bayesian inference of phylogenetic trees. Bioinformatics. 2001;17:754-5.

33. Bandelt $\mathrm{H}$, Forster $\mathrm{P}$, Rohl A. Median-joining networks for inferring intraspecific phylogenies. Mol Biol Evol. 1999;16:37-48.

34. Garrood WT, Zimmer CT, Gutbrod O, Lüke B, Williamson MS, Bass C, et al. Influence of the RDL A301S mutation in the brown planthopper Nilaparvata lugens on the activity of phenylpyrazole insecticides. Pestic Biochem Physiol. 2017;1:1-8.

35. Wang Y, Wang M. Factors affecting the outbreak and management tactics of brown planthopper in China recent years. Pestic Sci Adm. 2007:25:49-54

36. Kang S, Jung J, Lee S, Hwang H, Kim W. The polymorphism and the geographical distribution of the knockdown resistance ( $k d r)$ of Anopheles sinensis in the Republic of Korea. Malar J. 2012;11:151. 